\title{
Cleokurape
}

Nica 'oo

\section{Nonlinear polarisation effects in fibre Bragg gratings: experiment and theory}

N. G. R. Broderick ${ }^{1}$, G. D'Alessandro ${ }^{2}$, A. D Fitt ${ }^{2}$, M. Ibsen $^{1}$, T. M. Monro ${ }^{1}$, D. J. Richardson ${ }^{1}$ and D. Taverner ${ }^{1}$ 1: Optoelectronics Research Centre, University of Southampton, Southampton, SO17 1BJ, UK.

Phone: +44(0)1703 593144, Fax: +44(0)1703 593142, email: ngb@orc.soton.ac.uk

2: Faculty of Mathematical Studies, University of Southampton, Southampton SO17 1BJ, UK. Phone: +44 (0)1703 595141, Fax: $+44(0) 1703$ 595147, email: adf@maths.soton.ac.uk

Using a $8 \mathrm{~cm}$ long apodised fibre Bragg grating we have examined nonlinear polarisation rotation for pulses inside the photonic bandgap. In addition we have developed a theoretical model to simulate the system and good agreement between theory and experiment has been obtained. 


\title{
Nonlinear polarisation effects in fibre Bragg gratings: experiment and theory
}

\author{
N. G. R. Broderick ${ }^{1}$, G. D'Alessandro ${ }^{2}$, A. D Fitt ${ }^{2}$, M. Ibsen $^{1}$, T. M. Monro ${ }^{1}$, D. J. Richardson ${ }^{1}$ and D. Taverner ${ }^{1}$ \\ 1) Optoelectronics Research Centre, University of Southampton, Southampton, SO17 1BJ, UK. Phone: +44 (0)1703 593144, \\ Fax: $+44(0) 1703$ 593142, email: ngb@orc.soton.ac.uk \\ 2) Faculty of Mathematical Studies, University of Southampton, Southampton SO17 1BJ, UK
}

Recently we demonstrated the operation of an all-fibre nonlinear AND gate based on polarisation coupled gap solitons [1]. In this system when a single pulse was incident upon the grating it was reflected irrespective of its polarisation. However when two pulses with orthogonal polarisations were incident upon the grating a coupled gap soliton was formed which was able to propagate through the grating [2].

In addition to the AND gate we also looked at the polarisation dynamics of a single linearly polarised pulse propagating in a fibre Bragg grating. Briefly we used high nanosecond pulses obtained from a sequence of cascaded fibre amplifiers (as in Ref[1]). These pulses were launched into a $8 \mathrm{~cm}$ apodised fibre Bragg grating and at the output light from the two orthogonal polarisations were directed onto a fibre coupled photodiode and a sampling oscilloscope with a time resolution of $\sim 50 \mathrm{ps}$. These results are shown in Fig. 1(a) for both the input (solid line) and cross polarisations (dashed line). In these figures the initial peak is due to the intrinsic chirp on the leading edge of the pulse which is broader than the grating and is present in all traces regardless of the intensity. The other peaks are due to the formation of gap solitons inside the grating. What is clearly apparent from Fig. 1(a) is that the solitons have preferentially coupled to the orthogonal polarisation compared to the chirped peak.

To understand these results we have modeled the system using a set of nonlinear coupled mode equations which were solved numerically to model the experiment. These results are shown in Fig. 1(b) in which the parameters match as closely as possible the actual experimental parameters. Note that as in the experimental results the soliton components are preferentially rotated compared to the non soliton components.

In conclusion we have successfully modeled the effect of nonlinear polarisation rotation in fibre Bragg gratings. In the future we expect the model to be used to optimise the operation of nonlinear logic gates based on polarisation coupled gap solitons.

[1] D. Taverner et al. Opt. Lett. 23(4) pp259-261 (1998)

[2] C. M. de Sterke and J. E. Sipe, in Progress in Optics, edited by E. Wolf (North Holland, Amsterdam, 1994), Vol. XXXIII, Chap. III Gap Solitons, pp. 203-260.
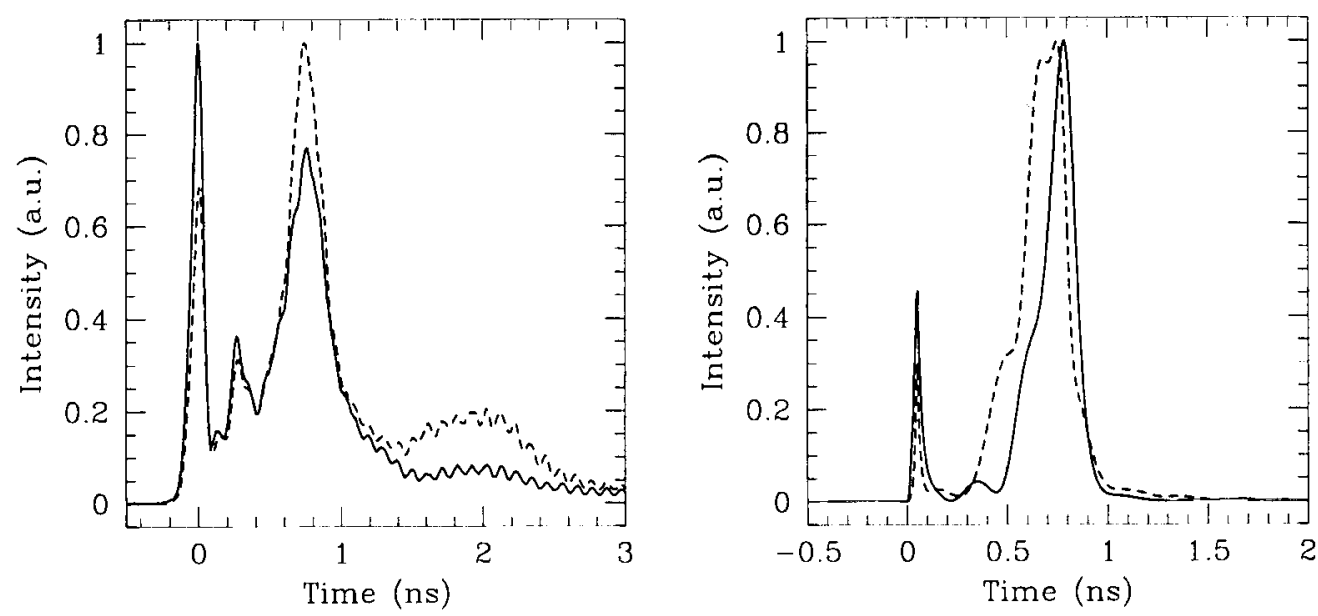

FIG. 1. Output for both the input (solid line) and orthogonal polarisations (dashed line). The graph on the left is the experimental curve while the one on the right is the theoretical curve. All curves have been scaled so that the maximum intensity is unity. 